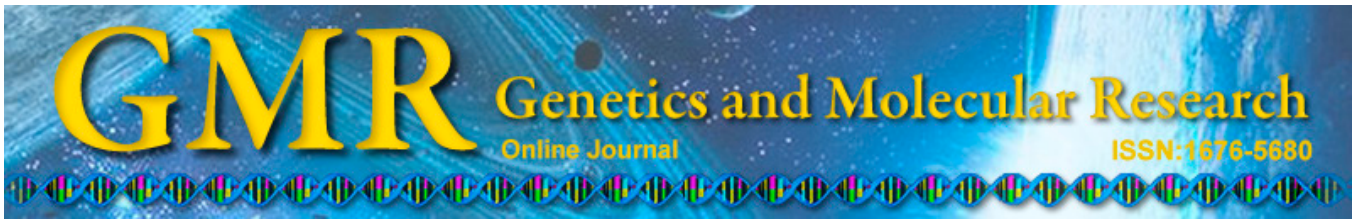

\title{
Silencing FKBP38 gene by siRNA induces activation of $m$ TOR signaling in goat fetal fibroblasts
}

\author{
Y.T. Fu*, X. Zheng*, Q. He, X.Y. Jia, Z.X. Guo, R.Y. Yao, Y.F. Wang and \\ Z.G. Wang \\ College of Life Sciences, Inner Mongolia University, Hohhot, China \\ *These authors contributed equally to this study. \\ Corresponding authors: Z.G. Wang / Y.F. Wang \\ E-mail: 1swzg@imu.edu.cn / wyf-imu@163.com
}

Genet. Mol. Res. 14 (3): 9675-9682 (2015)

Received February 10, 2015

Accepted May 15, 2015

Published August 14, 2015

DOI http://dx.doi.org/10.4238/2015.August.14.30

\begin{abstract}
FKBP38 (also known as FKBP8) is a unique member of the FK506-binding protein (FKBP) family, and its role is controversial because it acts as an upstream regulator of the mTOR signaling pathway, which controls cell growth, proliferation, and differentiation. This study aimed to explore the role of FKBP38 in the activation of mTOR signaling in Cashmere goat (Capra hircus) fetal fibroblasts. To construct a Cashmere goat FKBP38 siRNA eukaryotic expression vector that targets FKBP38 mRNA, we designed shRNA based on the gene sequence deposited in GenBank (accession No. JF714970) and synthesized a DNA fragment encoding the shRNA. The DNA fragment was inserted into the pRNAT-U6.1/Neo vector to construct an expression vector of shRNA, which was labeled pRNAT-FKBP38-shRNA. The recombinant plasmid was used to transfect Cashmere goat fetal fibroblasts $(\mathrm{GFb})$ using lipofectamine ${ }^{\mathrm{TM}} 2000$. We found that cells were successfully transfected with pRNAT-U6.1/Neo-FKBP38-shRNA. Green fluorescence could be observed in cells following 48-h transfection. Proteins were then isolated from GFbs transfected with pRNAT-FKBP38-shRNA and from control
\end{abstract}


cells, and protein expression was analyzed by western blot. Expression of FKBP38 decreased and mTOR signaling was activated, which induced the phosphorylation of mTOR, S6, and 4EBP1. Thus, FKBP38 genesilencing activates mTOR signaling in goat cells.

Key words: FKBP38, Short hairpin RNA; mTOR; Fetal fibroblasts; Inner Mongolia Cashmere goat

\section{INTRODUCTION}

A new member of the FK506-binding protein (FKBP) family, FKBP38 (also known as FKBP8), was isolated from a cDNA library of human T-cells in the early 1990s (Lam et al., 1995). FKBP38 is a unique member of the FKBP family, and is membrane-anchored, lies in the membranes of mitochondria and the endoplasmic reticulum (ER), and mediates the biogenesis of membrane proteins on the cytoplasmic side of the ER membrane (BanasavadiSiddegowda et al., 2011). FKBP38 contains FKBP_C, TPR, and TM domains. An interaction between the TPR domain of FKBP38 and the S4 subunit of 26S proteasome enables FKBP38 to anchor the $26 \mathrm{~S}$ proteasome to organellar membranes (Nakagawa et al., 2007). With the help of the TM domain, FKBP38 can bind to Bcl-2 through a charge-sensitive loop (Haupt et al., 2012) to control apoptosis, and $\mathrm{Ca}^{2+}$ modulates the interaction between the FKBP38 catalytic domain and Bcl-2 (Maestre-Martinez et al., 2011). FKBP38 is associated with many cell processes, including protein synthesis, cell proliferation and apoptosis, and FKBP38 ${ }^{-/}$mice die soon after birth (Shirane et al., 2008).

FKBP38 was identified as an endogenous inhibitor of the mammalian target of rapamycin complex 1 (mTORC1) in vitro and in vivo. Under conditions of amino acid or serum starvation, this mTOR inhibitor binds to mTOR via its FKBP_C domain and interferes with mTOR function in a similar manner to the FKBP12-rapamycin complex (Bai et al., 2007). Under conditions rich in growth factors and nutrients, the FKBP_C domain might interact with Rheb-GTP to activate downstream mTOR signaling by releasing mTOR from FKBP38 (Bai et al., 2007; Proud et al., 2007).

Rheb is an upstream regulatory factor in the mTOR signaling pathway, which contains a RAS domain, including switch I and switch II regions. The switch I region has been shown to interact with the FKBP_C domain of FKBP38 and displace it from mTORC1 in cells (Maestre-Martínez et al., 2006; Ma et al., 2008). Otherwise, overexpression or knockdown (Wang et al., 2008; Maehama et al., 2008) of FKBP38 does not affect the phosphorylation of mTORC1 substrates. Subsequent studies reported that FKBP38 is not involved in the Rheb-dependent activation of mTORC1 in vitro (Sato et al., 2009). These discrepancies indicate that further research is needed to verify the key role of FKBP38 in the regulation of mTOR signaling.

In the present study, to characterize the role of FKBP38 in mTOR signaling in goat cells, we designed a shRNA based on the sequence deposited in GenBank (accession No. JF714970) and synthesized a DNA fragment encoding the shRNA. The DNA fragment was inserted into a pRNAT-U6.1/Neo vector to construct an expression vector containing shRNA, and the recombinant plasmid was transfected into GFbs, which then expressed siRNA targeting FKBP38 mRNA. FKBP38 gene-silencing induced phosphorylation of mTOR, S6, and 4EBP1. Thus, silencing of the FKBP38 gene activates mTOR signaling in goat cells. FKBP38 is functional in goat fetal fibroblasts and regulates mTOR signaling. 


\section{MATERIAL AND METHODS}

\section{Cell culture conditions}

Inner Mongolia Cashmere goat fetal fibroblasts $(\mathrm{GFb})$ were maintained as monolayer cultures in Dulbecco modified Eagle's medium (DMEM)/F12 (Gibco, Paisley, PA49RF, Scotland, UK) supplemented with $10 \%$ fetal bovine serum (FBS, Hyclone Laboratories, Inc. Logan, UT, USA) and penicillin/streptomycin (Sigma-Aldrich, Inc. St. Louis, MO, USA). Cell cultures were maintained and incubated at $37^{\circ} \mathrm{C}$ in humidified air with $5 \% \mathrm{CO}_{2}$.

\section{Antibodies}

Antibodies against phospho-S6 (Ser235/236), S6, and phospho-4EBP1 (Thr37/46) were purchased from Cell Signaling (Beverley, MA, USA). Antibodies against FKBP38, 4EBP1, phospho-mTOR (Ser2448), and mTOR were purchased from Abcam (Cambridge, UK). Anti- $\beta$-actin was obtained from Sigma Chemicals (St. Louis, MO, USA).

\section{Design of shRNA targeting FKBP38 and construction of a eukaryotic expression vector}

Small interfering RNA (siRNA) targeting the FKBP38 sequence and the relevant short hairpin RNA (shRNA) were designed (Table 1, Figure 1) based on the Cashmere goat FKBP38 nucleotide sequence (GenBank accession No. JF714970). A DNA fragment encoding the shRNA was synthesized and then inserted into pRNAT-U6.1/Neo vector to construct pRNAT-FKBP38-shRNA (Figure 2).

Table 1. siRNA sequences targeting FKBP38.

\begin{tabular}{llccccccc}
\hline No. & Sequence & Start & GC $\%$ & Scores & $\Delta$ E/Thermo dynamic & SNPs & Off-target & Pos-Motifs \\
\hline 1 & AAGGCGAGTATAGTGAGGCCA & 967 & 52.38 & 21.99 & $2.00 /-39.60$ & NA & $10 / 46$ & 0 \\
\hline
\end{tabular}

BamHI

HindIII

GGATCCCGGCGA GIATAGTGAGGCCATTCAAGAGAT GGCCT CACTATACTCGCCII IIITCCAARAGCTT

I Sense | Loop | Antisense

Termination Signal

Figure 1. Targeting shRNA sequences of the FKBP38 gene.

\section{In Vitro Transfection}

pRNAT-FKBP38-shRNA vectors were transfected into actively growing GFbs cultured on 6-well plates using lipofectamine ${ }^{\mathrm{TM}} 2000$ reagent (Invitrogen, Carlsbad, New Mexico, USA) according to the manufacturer instructions/pRNAT-U6.1/Neo that did not contain a specific short hairpin RNA was used as the control. The transfected cells were cultured under positive selection with G418 (Hyclone Laboratories, Inc. Logan, Utah, USA) for $48 \mathrm{~h}$ and analyzed by imaging with a digital fluorescent microscope (Olympus IX71). 


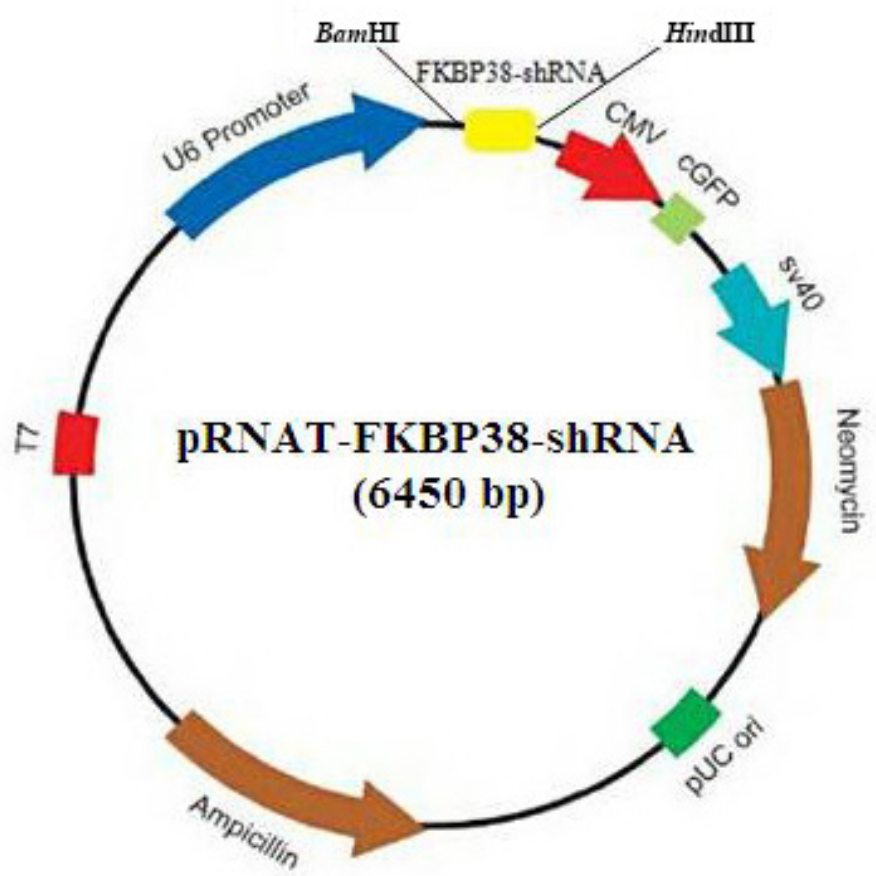

Figure 2. Schematic representation of pRNAT-FKBP38-shRNA.

\section{Analysis of the activity of mTOR signaling}

Transfected GFbs were harvested with trypsin, washed with cold phosphate-buffered saline, and lysed in cell lysis buffer. The cells were then placed on ice for $15 \mathrm{~min}$ and centrifuged at $13,000 \mathrm{rpm}$ at $4^{\circ} \mathrm{C}$ for $20 \mathrm{~min}$. The concentration of lysates was measured by Bradford assay (Bio-Rad Laboratories, USA). Equal amounts $(20 \mu \mathrm{g})$ of protein were electrophoresed on $10 \%(\mathrm{w} / \mathrm{v})$ sodium dodecyl sulfate polyacrylamide gels, transferred to polyvinylidene fluoride membranes, and incubated overnight with primary antibody overnight at $4^{\circ} \mathrm{C}$ and then peroxidase-conjugated secondary antibody at room temperature for $1 \mathrm{~h}$. Enhanced chemiluminescence (ECL) reagent (Amersham) Western Blotting System (GE Healthcare Bio-Sciences, Pittsburgh, PA, USA) was used to detect the signals. The resolved bands were quantified using Gel-Pro Analyzer 4.0 (Media Cybernetics, USA).

\section{RESULTS}

\section{Construction of the recombinant plasmid pRNAT-FKBP38-shRNA}

We designed shRNA and synthesized a 70-bp DNA fragment encoding the shRNA and ligated the DNA fragment into pRNAT-U6.1/Neo vector (6380 bp) to construct the recombinant expression vector pRNAT-FKBP38-shRNA (6450 bp). The recombinant plasmid was verified by restriction analysis (Figure 3 ) and electrophoresis. The plasmids were sequenced, and the shRNA gene was inserted downstream of U6 promoter. 


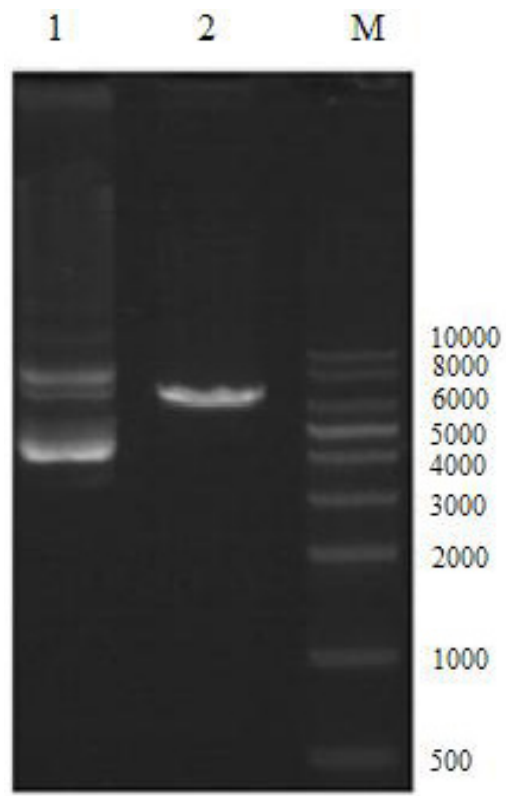

Figure 3. Restriction analysis of pRNAT-FKBP38-shRNA. Lane M: KB ladder; lane 1: pRNAT-FKBP38-shRNA plasmids; lane 2: pRNAT-FKBP38-shRNA plasmids digested by HindIII.

\section{Transfection of GFbs with the recombinant eukaryotic expression vector pRNAT-FKBP38-shRNA}

Lipofectamine $^{\mathrm{TM}} 2000$ was used to transfect GFbs with pRNAT-FKBP38-shRNA. pRNAT-U6.1/Neo and untransfected cells were used as control groups. The level of green fluorescence was examined under a fluorescent microscope (Figure 4), and images were obtained with a digital camera. Green fluorescence in the transfectants was observed following transfection for $48 \mathrm{~h}$.
A
B
$\mathrm{C}$

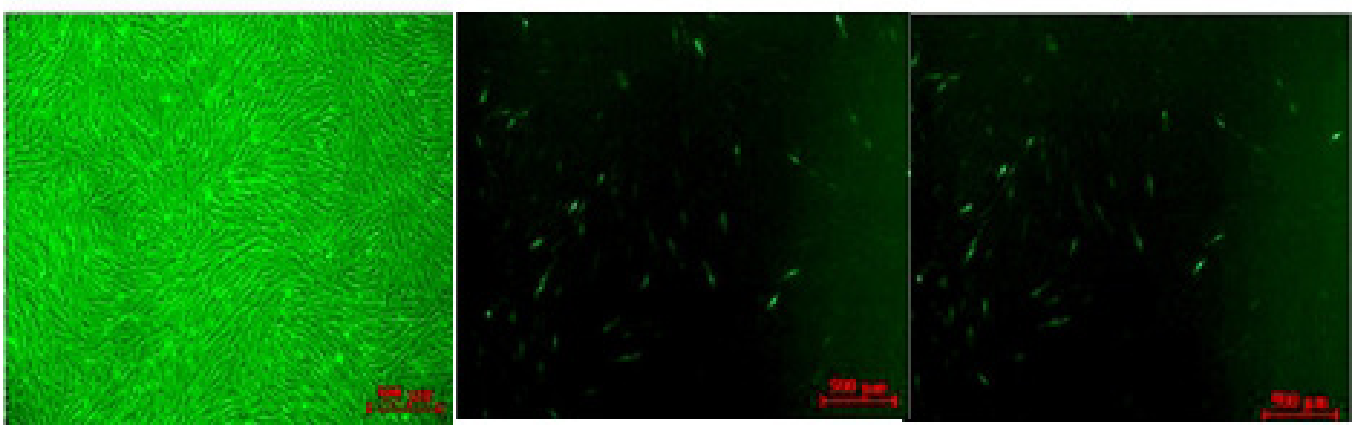

Figure 4. Cells expressing green fluorescence after transformation for $48 \mathrm{~h}$ (200X); (A) Bright-field images of control cells; (B) green fluorescence of cells transfected with pRNAT-U6.1/Neo; (C) green fluorescence of cells transfected with pRNAT-FKBP38-shRNA. 


\section{Analysis of FKBP38 expression and phosphorylation of mTOR, S6, and 4EBP1 in GFbs}

To detect expression of FKBP38 and activation of mTOR signaling in GFbs transfected with pRNAT-FKBP38-shRNA, proteins were isolated from transfected and control cells, and then analyzed by western blot. Results showed that expression of FKBP38 was decreased while phosphorylation of mTOR, S6, and 4EBP1 increased in the FKBP38 gene-silenced cells compared with the control cells (Figure 5). These data indicate that the FKBP38 gene was silenced and that mTOR signaling was activated, suggesting that FKBP38 is functional in GFbs and that it regulates mTOR signaling.
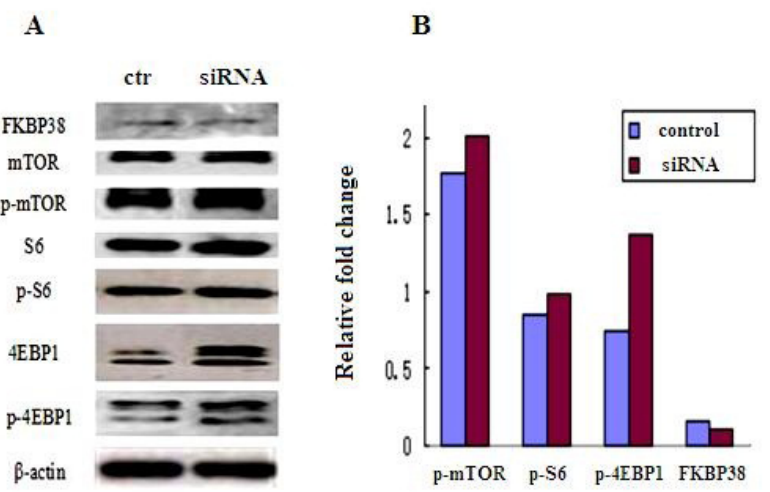

Figure 5. Western blot analysis of FKBP38, mTOR/phospho-mTOR (Ser2448), S6/ phospho-S6 (Ser235/236), and 4EBP1/phospho-4EBP1 (Thr37/46) in goat fetal fibroblasts. (A) The expression of FKBP38 was decreased while phosphorylation of mTOR, S6, and 4EBP1 increased in the FKBP38 gene silenced cells compared with control cells; (B) The resolved bands were quantified.

\section{DISCUSSION}

RNA interference (RNAi) is an attractive tool used in cell culture, examination of living organs, and disease models, owing to its specific and robust suppression of targeted gene sequences (Sledz and Williams, 2005). RNAi is an endogenous mechanism of regulating gene expression via small interfering RNA (siRNA) or microRNA (miRNA), which promotes mRNA degradation in a highly sequence-specific manner (Fire et al., 1998; Dykxhoorn and Lieberman, 2006). It is a natural process through which double stranded (ds) RNA regulates specific gene activity and occurs in most eukaryotic cells. RNAi mediators encompass microRNAs (miRNAs) and small interference RNAs (siRNAs) (Ford and Toloue, 2010). Intensive investigation of RNAi has confirmed that it is an efficient experimental technique for use in the knockdown of specific genes.

pRNAT-U6.1/Neo is a GenScript siRNA expression vector designed for mammalian transfection. It carries a neomycin-resistance gene, which acts as a selectable marker and can be used to establish stable cell lines. This vector also contains a U6 promoter, which drives the expression of the shRNA in vector-based RNAi. CMV promoter is a strong promoter used for both in vitro and in vivo expression of proteins and the expression of GFP driven by it can be used to track the transfection efficiency (Ramanathan et al., 2005). pRNAT-U6.1/Neo has been widely used in RNAi (Nie et al., 2010). 
FK506-binding proteins (FKBPs) are members of the immunophilin family of proteins, which bind to the immunosuppressant drugs FK506 and rapamycin. FKBPs are multifunctional proteins that regulate the folding or export of other protein molecules as a result of their peptidyl-prolyl cis/trans isomerase (PPIase) activity (Shirane-Kitsuji and Nakayama, 2014). They are involved in many biological processes, including hormone signaling and stress responses. As a unique member of the FKBP family, FKBP38 plays a key role in various cellular processes. FKBP38 can suppress Sonic hedgehog (Shh) signaling in mouse embryos, which affects embryonic development (Saita et al., 2014). Additionally, FKBP38 can inhibit apoptosis by recruiting the anti-apoptotic proteins Bcl-2 and Bcl-xL to mitochondria. Mice deficient in FKBP38 die soon after birth, and manifest a defect in neural tube closure that results in part from unrestrained apoptosis (Shirane et al., 2008). A recent report suggested that both $\mathrm{Hsp} 90$ and the non-structural protein 5A (NS5A) of the hepatitis $\mathrm{C}$ virus (HCV) interact specifically with FKBP38 through its TPR domain, and the ternary complex formation plays a critical role in HCV RNA replication (Tani et al., 2013).

As an endogenous inhibitor, FKBP38 impairs the activation of mTORC1, and this inhibition can be reversed with the combination of Rheb with FKBP38 (Bai et al., 2007). In previous studies, we have confirmed the interactions between Rheb and FKBP38 experimentally in goat cells (Wang et al., 2014). In the present study, we showed that KBP38 gene-silencing induced phosphorylation of mTOR, S6, and 4EBP1. FKBP38 is functional in GFbs and regulates mTOR signaling. Our data suggest that FKBP38 is a negative regulator upstream of mTORC1.

\section{CONCLUSIONS}

In conclusion, a shRNA eukaryotic expression vector targeting Cashmere goat FKBP38 mRNA was successfully constructed, and goat fetal fibroblasts were transfected with the recombinant pRNAT-FKBP38-shRNA vector, and transgenic cells expressing green fluorescence were obtained. In addition, the phosphorylation of mTOR, S6, and 4EBP1 was increased in transfected cells. FKBP38 gene-silencing activates mTOR signaling and FKBP38 is negative upstream regulator of $\mathrm{mTORC} 1$ in goat cells.

\section{Conflicts of interest}

The authors declare no conflict of interest.

\section{ACKNOWLEDGMENTS}

Research supported by grants from the Natural Sciences Foundation of China (\#31160469 and \#31360561) and a Graduate Student Research Project of the Inner Mongolia University, and Major Projects for New Varieties of Genetically Modified Organisms (\#2014ZX08008-002).

\section{REFERENCES}

Bai X, Ma D, Liu A, Shen X, et al. (2007). Rheb activates mTOR by antagonizing its endogenous inhibitor, FKBP38. Science 318: 977-980.

Banasavadi-Siddegowda YK, Mai J, Fan Y, Bhattacharya S, et al. (2011). FKBP38 peptidylprolyl isomerase promotes the folding of cystic fibrosis transmembrane conductance regulator in the endoplasmic reticulum. J. Biol. Chem. 286: 43071-43080. 
Dykxhoorn DM and Lieberman J (2006). Running interference: Prospects and obstacles to using small interfering RNAs as small molecule drugs. Annu Rev. Biomed. Eng. 8: 377-402.

Fire A, Xu S, Montgomery MK, Kostas SA, et al. (1998). Potent and specific genetic interference by double-stranded RNA in Caenorhabditis elegans. Nature 391: 806-811.

Ford LP and Toloue MM (2010). Delivery of RNAi mediators. Wiley Interdiscip. Rev. RNA 1: 341-350.

Haupt K, Jahreis G, Linnert M, Maestre-Martinez M, et al. (2012). The FKBP38 catalytic domain binds to Bcl-2 via a charge-sensitive loop. J. Biol. Chem. 287: 19665-19673.

Lam E, Martin M and Wiederrecht G (1995). Isolation of a cDNA encoding a novel human FK506-binding protein homolog containing leucine zipper and tetratricopeptide repeat motifs. Gene 160: 297-302.

Ma D, Bai X, Guo S and Jiang Y (2008). The switch I region of Rheb is critical for its interaction with FKBP38. J. Biol. Chem. 283: 25963-25970.

Maehama T, Tanaka M, Nishina H, Murakami M, et al. (2008). RalA functions as an indispensable signal mediator for the nutrient-sensing system. J. Biol. Chem. 283: 35053-35059.

Maestre-Martínez M, Edlich F, Jarczowski F, Weiwad M, et al. (2006). Solution structure of the FK506-binding domain of human FKBP38. J. Biomol. NMR 34: 197-202.

Maestre-Martinez M, Haupt K, Edlich F, Neumann P, et al. (2011). A charge-sensitive loop in the FKBP38 catalytic domain modulates Bcl-2 binding. J. Mol. Recognit. 24: 23-34.

Nakagawa T, Shirane M, Iemura S, Natsume T, et al. (2007). Anchoring of the $26 \mathrm{~S}$ proteasome to the organellar membrane by FKBP38. Genes Cells 12: 709-719.

Nie L, Das Thakur M, Wang Y, Su Q, et al. (2010). Regulation of U6 promoter activity by transcriptional interference in viral vector-based RNAi. Genomics Proteomics Bioinformatics 8:170-179.

Proud CG (2007). Cell signaling. mTOR, unleashed. Science 318: 926-927.

Ramanathan M, Haskó G and Leibovich SJ (2005). Analysis of signal transduction pathways in macrophages using expression vectors with CMV promoters: a cautionary tale. Inflammation 29: 94-102.

Saita S, Shirane M, Ishitani T, Shimizu N, et al. (2014). Role of the ANKMY2-FKBP38 axis in regulation of the Sonic hedgehog (Shh) signaling pathway. J. Biol. Chem. 289: 25639-25654.

Sato T, Nakashima A, Guo L and Tamanoi F (2009). Specific activation of mTORC1 by Rheb G-protein in vitro involves enhanced recruitment of its substrate protein. J. Biol. Chem. 284: 12783-12791.

Shirane-Kitsuji M and Nakayama KI (2014). Mitochondria: FKBP38 and mitochondrial degradation. Int. J. Biochem. Cell Biol. 51: 19-22.

Shirane M, Ogawa M, Motoyama J and Nakayama KI (2008). Regulation of apoptosis and neurite extension by FKBP38 is required for neural tube formation in the mouse. Genes Cells 13: 635-651.

Sledz CA and Williams BR (2005). RNA interference in biology and disease. Blood 106: 787-794.

Tani J, Shimamoto S, Mori K, Kato N, et al. (2013). Ca(2+)/S100 proteins regulate HCV virus NS5A-FKBP8/FKBP38 interaction and HCV virus RNA replication. Liver Int. 33: 1008-1018.

Wang X, Fonseca BD, Tang H, Liu R, et al. (2008). Re-evaluating the roles of proposed modulators of mammalian target of rapamycin complex 1 (mTORC1) signaling. J. Biol. Chem. 283: 30482-30492.

Wang X, Wang Y, Zheng X, Hao X, et al. (2014). Direct Interaction between Ras Homolog Enriched in Brain and FK506 Binding Protein 38 in Cashmere Goat Fetal Fibroblast Cells. Asian-Aust. J. Anim. Sci. 27: 1671-1677. 